\title{
Sub-kHz Quantum Linewidth Semiconductor Laser On Silicon Chip
}

\author{
Christos T. Santis, Yaakov Vilenchik, and Amnon Yariv \\ California Institute of Technology, M/C 128-95, Pasadena, California 91125 \\ christos@caltech.edu \\ Naresh Satyan, and George Rakuljic \\ Telaris Inc., Santa Monica, California 90403
}

\begin{abstract}
We report on a semiconductor laser on silicon platform with record-low sub-kHz quantum noise-limited linewidth, based on spontaneous emission control and optical loss reduction.

OCIS codes: (140.3430) Laser theory; (140.3490) Lasers, distributed-feedback; (140.5960) Semiconductor lasers
\end{abstract}

\section{Introduction}

The coherence of laser light is quickly becoming a performance-limiting factor in the growing fields of high data rate communications, phase-sensitive sensing and metrology, and high-precision time/frequency division. These areas have thus far relied on fiber and external cavity diode lasers for the requisite coherence, yet as the everincreasing demand for performance is coupled with a premium on cost and size, more scalable solutions are required. The distributed feedback semiconductor laser (DFB-SCL, [1]), which has powered the worldwide fiber optic network for over four decades possesses a coherence which is limited to levels around a MHz, a value which falls short of that required for modern phase-sensitive applications. We show that the present canonical design of the SCL is fundamentally injurious to high coherence. We present and experimentally demonstrate a new design paradigm that fundamentally overcomes the limitations of the conventional design and delivers up to three orders of magnitude improvement in coherence.

\section{Theory and design}

The main coherence corrupting mechanism is phase fluctuations due to photons of random phase, which are emitted into the laser mode, as a result of the spontaneous recombination of injected electrons and holes in the active region. Under the effect of a large number of spontaneous emission events over a time $\tau$, the laser field undergoes a random walk in phase space with a variance given by [2]

$$
\left\langle\Delta \theta(\tau)^{2}\right\rangle=\frac{R_{s p}}{2 n_{\ell}} \tau=\frac{W_{s p N_{2 t}}}{2 n_{\ell}} \tau,
$$

where $W_{s p}$ is the spontaneous emission rate $\left(s^{-1}\right)$ into the lasing mode, $N_{2 t}$ the absolute number of excited carriers (clamped at its threshold value), and $n_{l}$ the number of coherent quanta in the mode. These are the three fundamental factors that partake in setting the quantum limit of a laser's coherence. For the case of a semiconductor laser, equation (1) can be recast as a function of macroscopically controlled parameters:

$$
(\Delta v)_{S-T}=\frac{3}{2 \pi} \frac{e(c / n)^{3}}{\eta_{i}\left(I-I_{t h}\right) \omega_{\ell} \tau_{s p} \Delta v_{a}}\left(\frac{\left|\bar{E}_{\ell}\left(\bar{r}_{a}\right)\right|^{2}}{Q} N_{t r}+\frac{\omega_{\ell}}{g^{\prime} Q^{2}}\right)
$$

where $\tau_{s p}$ is the excited carrier lifetime due to spontaneous emission into all modes of the laser cavity, $\Delta v_{a}$ the spontaneous emission (gain) linewidth of the active medium, $N_{t r}$ the excited carrier population necessary to reach transparency, and $g^{\prime}$ the gain coefficient $\left(s^{-1}\right) .\left|\bar{E}_{\ell}\left(\bar{r}_{a}\right)\right|^{2}$ is the normalized modal energy intensity of the lasing mode at the location of the active medium, whereas $Q$ is the loaded quality factor of the mode, measure of the photon lifetime. According to equation (2), there are two components of spontaneous emission into the laser mode, one due to the transparency population and one due to the additional population required to overcome optical losses. For typical SCL numbers $\left(Q \sim 10^{4}\right)$, the two terms of (2) are of roughly comparable magnitudes, thus highlighting the importance of suppressing both of them. Increasing $Q$, if possible, would result in the transparency term becoming the limiting one. Thus, the most effective scheme would comprise simultaneous control over $\left|\bar{E}_{\ell}\left(\bar{r}_{a}\right)\right|^{2}$ and $Q$. Modal losses, and hence $Q$, in a conventional SCL are limited by absorption (i.e. free-carrier, intervalence-band) in the active region, as well as in the heavily doped contact regions. Removal of modal energy from the III-V material, a major departure from the canonical design that has the mode residing exclusively in the lossy semiconductor medium (fig 1(b)), is thus mandated.

We exploit the optical design flexibility afforded by the hybrid Si/III-V platform to remove modal energy from the active medium and store it in the low-loss silicon, thus increasing the Q factor of the laser mode. This was most 
prominently demonstrated on a Si/III-V platform (fig. 1(a)), where a silicon resonator with a quality factor of $Q_{S i} \sim 10^{6}$ as an integral element of the laser cavity, was shown to create a dramatic potential for loss reduction [3]. Furthermore and importantly, we introduce a spacer layer of low refractive index (e.g. $\left.\mathrm{SiO}_{2}\right)$ between the III-V and silicon to directly control the rate of spontaneous emission into the lasing mode through manipulation of $\left|\bar{E}_{\ell}\left(\bar{r}_{a}\right)\right|^{2}$. Increasing the thickness of the spacer layer suppresses the spontaneous emission contributed by the transparency term via $\left|\bar{E}_{\ell}\left(\bar{r}_{a}\right)\right|^{2}$, while at the same time increasing $Q$, due to the decreasing overlap of the mode with the lossy III$\mathrm{V}$ region (figs. $1(\mathrm{~b}, \mathrm{c})$ ).

\section{Results}

We fabricated and measured spacer hybrid Si/III-V lasers with varying spacer thickness. The frequency noise spectral density for three lasers with spacer thickness of $30 \mathrm{~nm}, 100 \mathrm{~nm}$, and $150 \mathrm{~nm}$ are shown in fig. 1(d). All lasers were measured at the same offset from threshold $\left(I-I_{t h}\right)$, to ensure that linewidth reduction due to current did not enter in the comparison. The quantum noise-limited linewidth, extracted at the white noise level $(\sim 300$ $\mathrm{MHz}$ ), decreases from nearly $40 \mathrm{kHz}$ for the $30 \mathrm{~nm}$ spacer to almost $1 \mathrm{kHz}$ for the $150 \mathrm{~nm}$ one. A noise floor, set by phase noise injected by the EDFA used to amplify the laser signal, limits the measurement of the thickest spacer hybrid. This is evident in the noise rise at higher frequencies and was found to vary with the amount of amplification. At the lowest point $(\sim 1 \mathrm{kHz})$, the laser and amplifier contribute approximately equal noise, hence putting the intrinsic laser linewidth well below the $1 \mathrm{kHz}$ mark. This is, to the best of our knowledge, the smallest quantum linewidth ever measured for a free-running semiconductor laser. The black trace in fig. 1(d) corresponds to a control laser (JDSU DFB), with a minimum linewidth of $100 \mathrm{kHz}$ at $\sim 10 \mathrm{x}$ higher pump level than the one used for the hybrid lasers. At the same pump offset as the spacer hybrid lasers, the control laser exhibits a linewidth of nearly $1 \mathrm{MHz}$, thus a linewidth reduction of nearly three orders of magnitude is achieved for roughly the same input power. In the $150 \mathrm{~nm}$ spacer hybrid, about $1.5 \%$ of the mode's energy resides in the III-V, with $0.2 \%$ in the QW region, while more than $95 \%$ is confined in silicon.

(a)

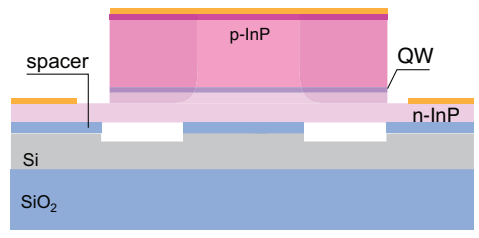

(b)

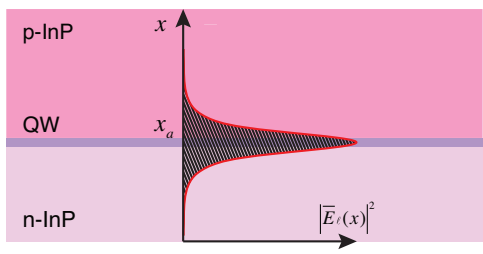

(c)

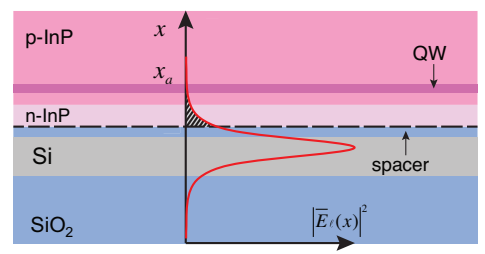

(d)

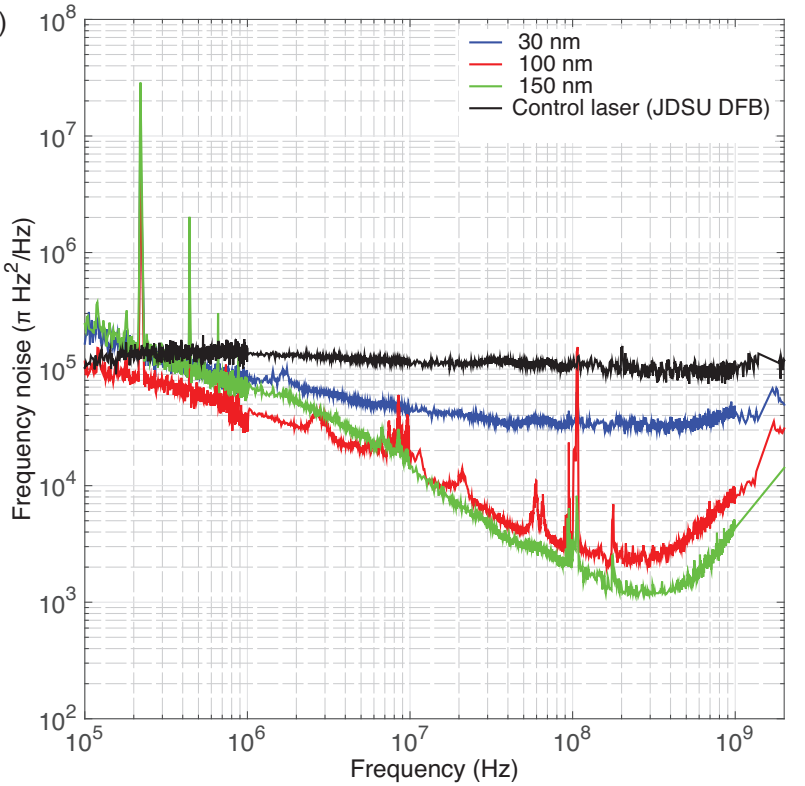

Fig. 1. (a) Cross-section of a hybrid Si/III-V laser with embedded spacer layer for spontaneous emission control, (b) transverse field profile of a conventional monolithic III-V laser design, (c) transverse field profile of a spacer hybrid Si/III-V laser, (d) frequency noise spectral density for three spacer hybrid lasers and control laser (all spacer lasers measured at $30 \mathrm{~mA}$ from threshold, control laser measured at $275 \mathrm{~mA}$ ).

\section{References}

[1] M. Nakamura, A. Yariv, H. W. Yen, S. Somekh, and H. L. Garvin, "Optically pumped GaAs surface laser with corrugation feedback," Appl. Phys. Lett., vol. 22, pp. 515-516, 1973.

[2] A. L. Schawlow and C. H. Townes, "Infrared and optical masers," Phys. Rev., vol. 112, pp. 1940-1949, 1958.

[3] C. T. Santis, S. T. Steger, Y. Vilenchik, A. Vasilyev, and A. Yariv, "High-coherence semiconductor lasers based on integral high-Q resonators in hybrid Si/III-V platforms," Proceedings of the National Academy of Sciences of the United States of America, vol. 111, pp. 2879-2884, Feb 252014. 